\title{
Control de la hemoglobina sanguínea y el hematocrito en pacientes sometidos a lipoescultura y de miniabdominolipoplastía con los principios de la Técnica de Juarez Avelar.
}

\author{
Hemoglobin and hematocrit blood controls in patients treated with liposuction and \\ mini-abdominolipoplasty using juarez avelar principles techniques.
}

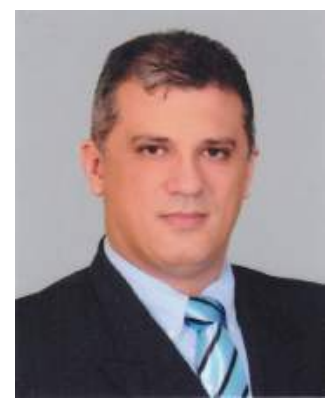

\begin{abstract}
Suárez Oyhamburú, D.*, Schaymann Lora, J. **
* Cirujano Plástico, Titular de la Sociedad Boliviana de Cirugía Plástica Estética y Reconstructiva (SBCPER), Titular de la FILACP, Clínica Niño Jesús Dos, Santa Cruz de la Sierra, Bolivia.

** Bioquímica Farmacéutica, MD. Coordinadora de Gestión Hospitalaria, Regente del Laboratorio de la Clínica Médica Siraní, Centro de Fertilización Asistida Clínica Siraní, Santa Cruz de la Sierra, Bolivia.
\end{abstract}

\section{Resumen}

Este es un estudio prospectivo, no comparativo, no experimental, longitudinal, de cohorte, que iniciamos en abril del 2008 hasta enero del 2015, de pacientes operados en mi consulta privada, empleando dos técnicas con principios similares como la lipoescultura y la mini abdominolipoplastía del Prof. Juárez Avelar. No consideramos a ningún paciente operado el año 2012 debido a la presencia en su mayoría de cirugías asociadas con otros procedimientos que no fueran única y exclusivamente las mencionadas.

Además debido a la afluencia en mayor cantidad de pacientes extranjeros lo cual dificultó la posibilidad de realizar los controles post operatorios en el mismo laboratorio.

El objetivo principal del presente trabajo fue medir y controlar la hemoglobina sanguínea y el hematocrito entre el zer y 5to día post operación y determinar hasta cuanto descienden estos valores por cada litro de grasa aspirada en los pacientes sometidos a estas técnicas. Así como también poder determinar el límite máximo aconsejable, esto expresado en litros, de la cantidad de grasa necesaria para extraer durante una liposucción teniendo en cuenta los valores de la hemoglobina sanguínea $(\mathrm{Hb})$ y el hematocrito(Hto) normal pre operatorios de los pacientes estudiados.
Todos sabemos que la determinación de la $\mathrm{Hb}$ y Hto en el post operatorio NO es un método exacto para definir el volumen a lipoaspirar sin embargo nos puede guiary ayudar a tener en cuenta el volumen aspirado de los posibles niveles de descenso de estos valores. El peso del paciente es el factor más importante en la estimativa a la hora de lipoaspirar y mantener hasta un $5 \%$ de límite en relación al peso corporal expresado en litro de grasa aspirada es fundamental y seguro para el paciente.

\section{Palabras claves:}

Lipoescultura, Mini abdominolipoplastía, Hemoglobina, Hematocrito.

\section{Abstract}

This is a prospective, no comparative, no experimental, longitudinal, and cohort study that started on April 2008 and continued through January 2015 . It was done with patients of my private practice using two similar principles such as liposculture and Juarez Avelar's technique known as mini abdominolipoplasty. We didn't consider any patient who had surgery done within the year 2012 since most of them had more than one procedure besides the ones mentions above. Also due to many incoming foreigns, we excluded them because it was difficult to have post surgical tests done at the same laboratory. 
The main idea of this present study was to measure and control the hemoglobin and hematocrit during the third and fifth day post surgical to determine how much these values descended for each liter of fat aspired in these patients who went through such procedures. It was also encourage to help determine the upper limit recommended , expressed in liters, of fat allowed to be aspired during a liposuction knowing ahead on time the pre- surgical values of hemoglobin $(\mathrm{Hb})$ and hematocrit $(\mathrm{Htc})$ for all the patients who took part of this study.

We all know that the $\mathrm{Hb}$ and $\mathrm{Htc}$ for post surgical is NOT an exact method to be used as a way to define the volume to lipo aspires. Instead, it can help us a lot as a guide for the amount of fat to aspire that can cause the values of these parameters to drop. The patient's weight is the most important factor for its safety and for the colleague to estimate the liposuction maintaining a limit up to $5 \%$ in relation to the body weight expressed in liters of fat extracted.

\section{Key words:}

Liposculture, mini abdominolipoplasty, hemoglobin, hematocrit.

\section{Introducción}

La lipoescultura es una técnica revolucionaria en la cirugía plástica que a partir de los años 80 cuando Illouz la desarrolló, marcó historia en el tratamiento y corrección de lipodistrofias del contorno corporal, contribuyendo también al mejoramiento estético del contorno abdominal. $(6,8)$.

Pero la mini abdominolipoplastía de Avelar es un procedimiento que trae nuevos conceptos... no se realiza despegamiento ni la resección del panículo cutáneo adiposo excedente, solo apenas la lipoaspiración de la camada profunda de la grasa o de la capa lamelar es decir por debajo de la fascia superficial. Así como tampoco no se realiza la plicatura de la musculatura, se reseca únicamente el colgajo cutáneo excedente, sin que exista lesión nerviosa, ni de los vasos perforantes y ni los comunicantes (arterias y venas), llamándola "Sistema Vascular Cerrado". (1, 2, 3).

En el área a retirarse el colgajo excedente de piel se realiza una lipoaspiración de la totalidad del panículo graso excedente, es decir en ambas camadas, avanzando más fácilmente el colgajo y de esta manera se preserva los vasos perforantes asegurando la buena nutrición del segmento abdominal inferior además al tener como principio la lipoaspiración, prácticamente no es cirugía sangrante. $(1,2,3)$.

Por este sencillo motivo es que incluimos en nuestro estudio esta técnica. El mini abdomen de Juárez Avelar se aplica en los casos de correcciones de cicatrices de abdomino plastias anteriores muy cómodamente y en los casos de lipodistrofias abdominales del abdomen inferior donde hay exceso de piel y no es necesario realizar plicaturas de la musculatura abdominal. $(1,2,3)$.

Dentro de los criterios para determinar el volumen de grasa total a lipoaspirar según la academia americana de cirugía cosmética tenemos:

1. Peso del paciente (hasta un $5 \%$ del peso corporal total expresado en litros.) factor principal.

2. Estado de salud general.

3. Total de superficie total a tratar.

4. Localización de las áreas a lipoaspirar.

5. Estimación de la cantidad de lidocaína y epinefrina que podemos usar.

6. Cirugías previas de lipoaspiración.(12,13)

Sin lugar a dudas que el factor "PESO" es el punto primordial a hora de determinar el límite máximo del volumen a lipoaspirar sin que existan riesgos de sangrados excesivos que ameriten transfusiones sanguíneas posteriores. (4, 7, 8,9 y13).

Si ejecutamos un ejercicio simple en un paciente de $70 \mathrm{ki}-$ los, y calculamos el $5 \%$ del peso corporal total expresado en litros, obtendremos que hasta 3,5 litros de grasa se pueda aspirar dentro de un margen probable de seguridad sin que exista la necesidad de hemo transfusiones.

\section{Objetivo principal.}

Determinar a través de la cuantificación de la hemoglobina sanguínea y el hematocrito en el post operatorio, hasta cuanto descienden estos valores por cada litro de grasa aspirada en los pacientes sometidos a lipo aspiración del contorno corporal y a mini abdominolipoplastía con la técnica de Juárez Avelar.

\section{Objetivos específicos.}

Cuantificar la Hemoglobina sanguínea y el Hematocrito en los pacientes sometidos a liposucción o a mini abdominolipoplastía de Juárez Avelar entre el zer o el 5to día de post operatorio.

Determinar el límite máximo "aconsejable” (expresado en litros) de la cantidad de grasa necesaria para extraer durante una liposucción teniendo en cuenta los límites de Hemoglobina sanguínea y Hematocrito normales pre operatorios de los pacientes estudiados.

\section{Material y métodos.}

Iniciamos este estudio en pacientes operados de mi consultorio privado a partir de abril del 2008 hasta enero del 2015. Nos vimos forzados por diferentes motivos a no considerar pacientes en el año 2012, tal vez por la gran o mayor afluen- 
cia de pacientes extranjeros en la consulta, por la dificultad de no poder realizar controles post operatorios en el mismo laboratorio y además por no encajarse dentro de los patrones de inclusión de este estudio así como a la ausencia de pacientes sometidos únicamente a estas dos técnicas pues realizamos cirugías asociadas a otros procedimientos de cirugía estética.

Incluimos en este estudio a los pacientes sometidos a Mini abdominolipoplastía con los principios del Prof. Juárez Avelar por considerar un procedimiento basado principalmente en la liposucción y por No representar una técnica agresiva que genere sangrado importante en el paciente ya que como manifestamos el fundamento básico de esta técnica es el sistema vascular cerrado con la preservación de vasos perforantes y la resección de la piel del colgajo.

Medimos y controlamos la hemoglobina sanguínea y el hematocrito entre el zer. y el 5to. día post operación por considerar que la hemodilución inducida por la administración de líquidos endovenosos durante la operación, la absorción de líquidos infiltrados durante el acto quirúrgico y el edema inmediato, podrían alterar los resultados en la cuantificación.

La Hemoglobina sanguínea fue cuantificada y medida a través de métodos de colorimetría automatizada y tabulación, así como micro centrifugación y formulación automatizada en el caso del hematocrito.

Todos los pacientes operados fueron sometidos a anestesia peridural con sedación endovenosa y pertenecían a la clasificación de riesgo quirúrgico pre operatorio ASA 1 y 2.

En todos los casos utilizamos $1000 \mathrm{mls}$ de suero fisiológico al o,9\% a temperatura ambiente o ligeramente pre calentado con 2 ampollas de adrenalina.

El volumen de líquidos infiltrados osciló entre $1000 \mathrm{mls}$ a $3500 \mathrm{mls}$ dependiendo de la cantidad estimada de grasa a lipoaspirary de las áreas corporales a trabajar.

En todos realizamos trombo profilaxis utilizando enoxiparina de 2omgs subcutáneo hasta la 6ta hora post operación aplicada en brazo derecho por la enfermera de piso y todos firmaron un consentimiento médico informado al momento de la internación.

También indicamos la rápida movilización de miembros inferiores en el lecho, evitar las fajas post operatorios modeladoras muy apretadas y la utilización de medias anti trombóticas automáticas intermitentes durante el acto quirúrgico.

Dentro de los “CRITERIOS DE EXCLUSIÓN “en este estudio descartamos los resultados de la hemoglobina y el hema- tocrito de los pacientes que hubiesen realizado el control post en diferentes laboratorios, esto para disminuir la posibilidad y el margen de error en la interpretación laboratorial. Punto importante lo que significó descartar varios pacientes sobre todo los extranjeros ya que no se encuadraron en el estudio.

Tampoco fueron parte de este estudio y no contabilizamos a los pacientes sometidos a otros procedimientos quirúrgicos adjuntos, es decir cirugías asociadas, que no fueran única y exclusivamente los dos procedimientos mencionados.

Descartamos también a pacientes con diabetes Mellitus, o portadores de enfermedades infectocontagiosas.

No tomamos en cuenta otras variables como la edad, sexo, raza, procedencia, cirugías previas de lipoescultura, áreas a lipoaspirar u otras técnicas de lipoaspiración que no fuera únicamente la convencional.

En la figura 1 y 2 respectivamente podemos apreciar algunos de los casos donde empleamos la técnica del mini abdomen con los principios de Juárez Avelar.
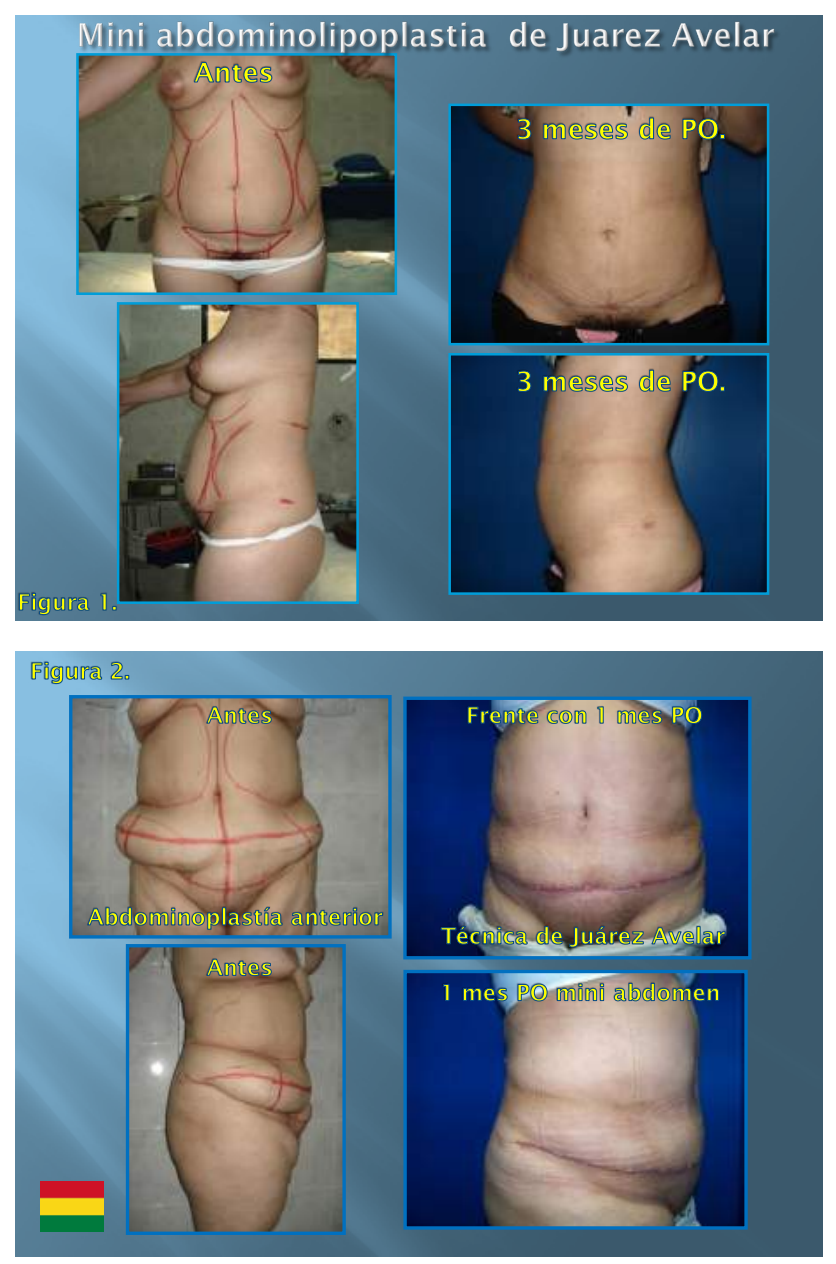
En la figura 3 apreciamos el desarrollo de la técnica de Juárez Avelar con el "sistema vascular cerrado " y la preservación vascular en el segmento inferior del abdomen.

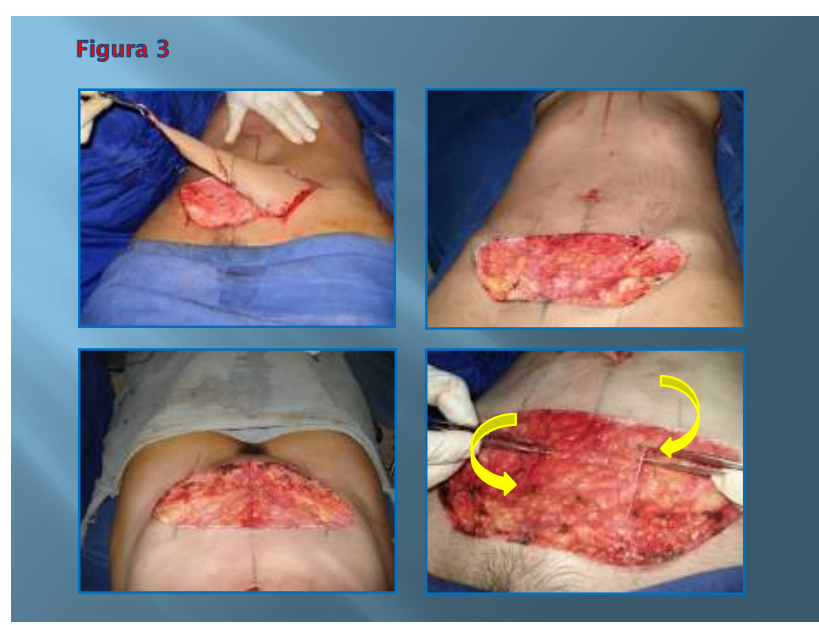

Figura 4 se observa a manera de ilustración un caso de lipoescultura del contorno corporal.

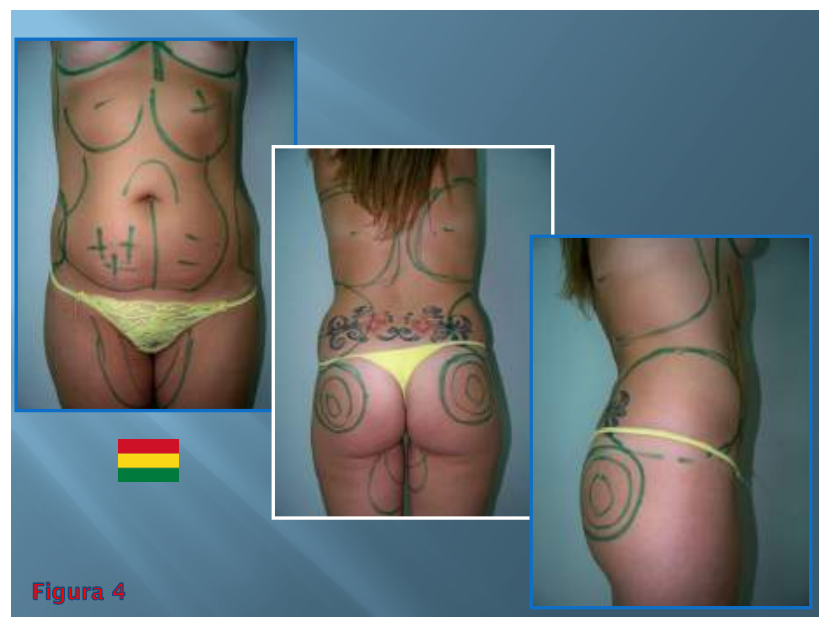

\section{Resultados.}

Fueron incluidos en este estudio a 73 pacientes desde abril del 2008 hasta enero del 2015 de los cuales 46 pacientes correspondían al grupo de lipoescultura del contorno corporal, cuyo valor promedio expresado en litros de grasa aspirada alcanzo 2210,43 mls por paciente.

Los otros 27 pacientes correspondían a mini abdominolipoplastía de Avelar, cuyo valor promedio expresado en litros de grasa aspirada alcanzo los 2335,92 mls por paciente.

El valor máximo aspirado fue de $4060 \mathrm{mls}$ y el mínimo fue de $850 \mathrm{mls}$. En el grupo del miniabdomen el valor máximo aspirado fue $3500 \mathrm{mls}$. Y el mínimo $1000 \mathrm{mls}$.

En la figura 5 observamos en esta gráfica, las líneas tendenciales de color negro de forma isoeléctrica sobre las líneas de color amarillo que muestran a los pacientes del grupo de lipoescultura, los valores del hematocrito PRE operatorios. Pero en las líneas de color Rojo la línea negra tiene una tendencia a descender a niveles por debajo de $30 \%$.

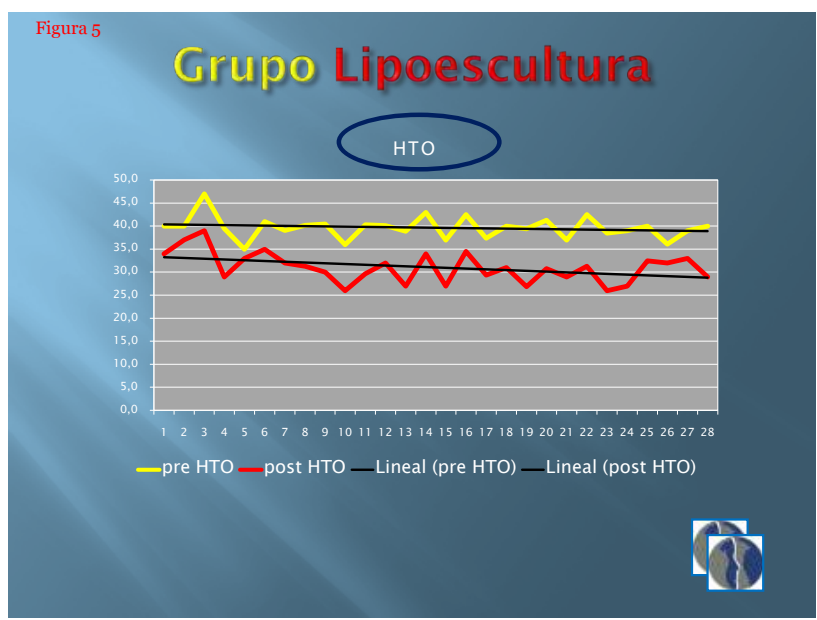

En la figura 6 observamos esta gráfica muy significativa que de alguna manera traduce lo mostrado por la gráfica 5 donde la línea negra sobre las gráficas de color rojo presenta un descenso y si esto lo traducimos en estas barras, observamos que para este grupo de lipoescultura, los valores del Hematocrito en el pre operatorio tenían un promedio de $39.7 \%$ pero sufre un descenso en el control post operatorio entre el zery 5 to día a $31.0 \%$

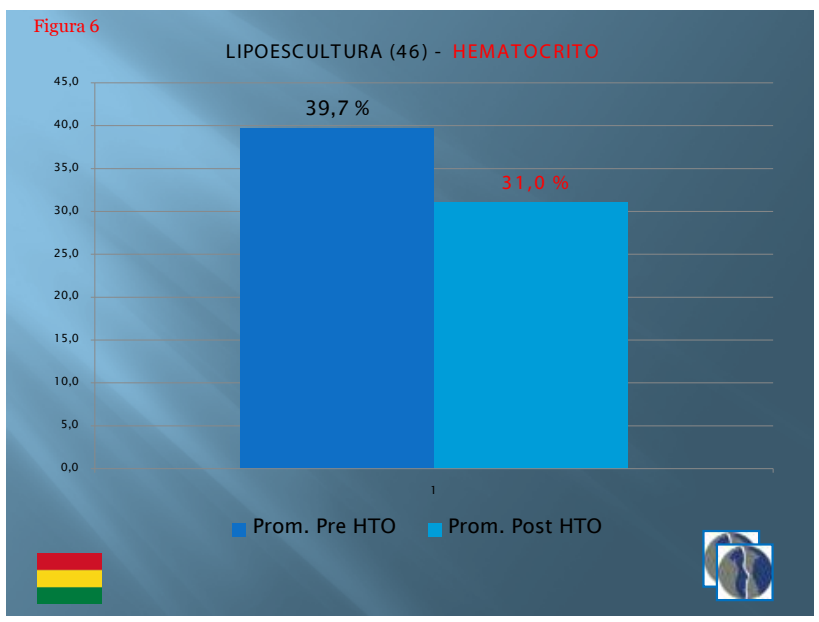

En la figura 7. podemos observar el comportamiento tendencial de la Hemoglobina en color negro sobre las líneas amarillas que al igual que el hematocrito muestra una línea isoeléctrica pero cuando realizamos el control post operatorio entre el zer y el 5to día vemos la línea de color negro sobre las líneas gráficas rojas cuya tendencia es descendente en el grupo post operatorio. 


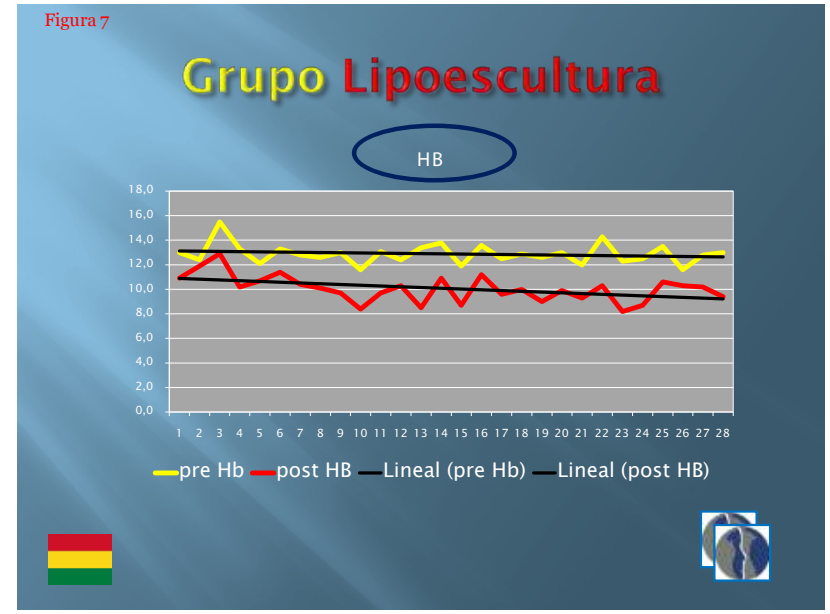

Pero si esto lo demostramos nuevamente en barras obtenemos mayor claridad al ver en la figura 8 para este grupo de lipoescultura controlando la hemoglobina que los niveles pre operatorios de hemoglobina alcanzaron un promedio de $12.9 \mathrm{grs} / \mathrm{dl}$, pero de color celeste más claro podemos observar la barra que nos muestra un descenso entre el zer y 5 to día a 10.1 grs /dls.

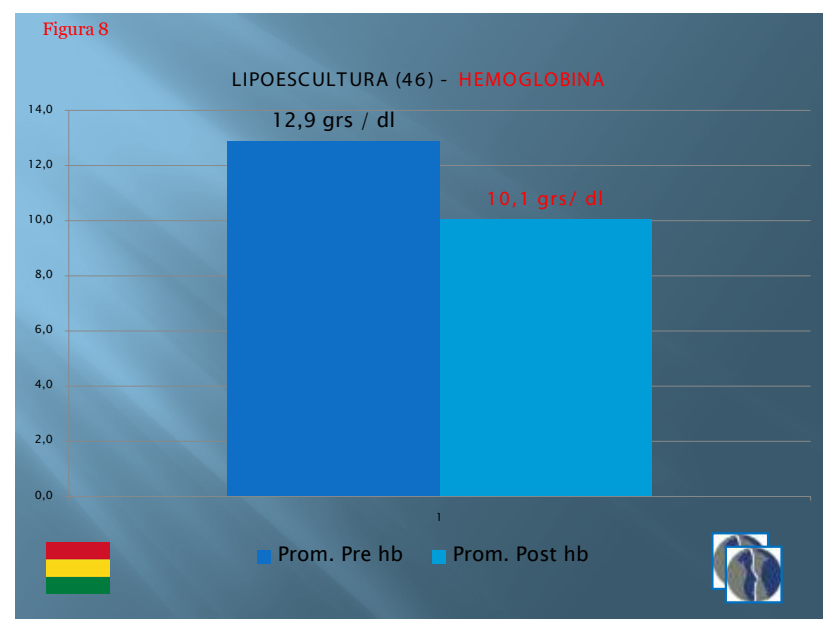

En la siguiente grafica que corresponde a la figura 9 podemos ver que la línea isoeléctrica tendencial de color rojo se repite para el grupo pre operatorio de los pacientes de mini abdomen pero en el control post operatorio del Hematocrito esta línea roja tendencial sobre las líneas gráficas verdes presenta un descenso que inicia a niveles por encima de $30 \%$ pero que baja a niveles cercanos a $29 \%$.

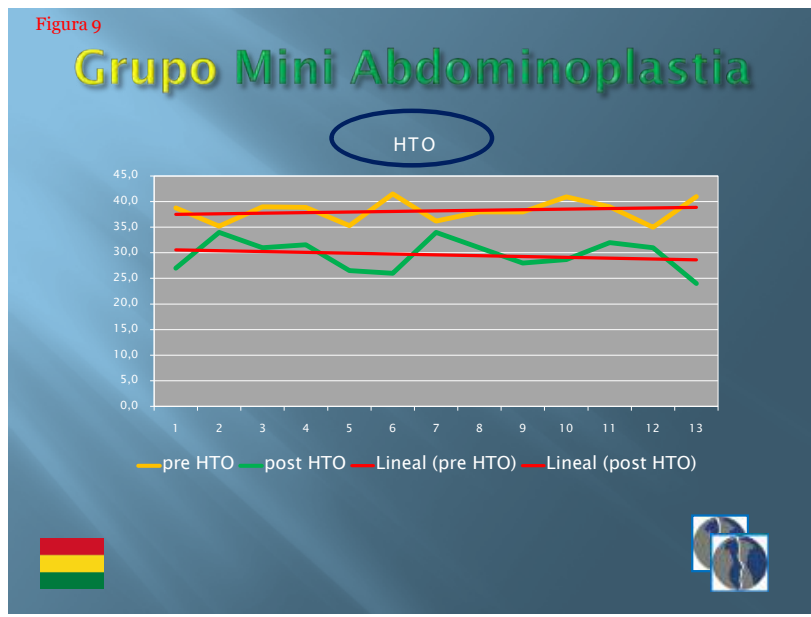

En la Figura 10, las barras son muy significativas y nos muestran el descenso de niveles pre operatorio del hematocrito de $38,2 \%$ a $29,6 \%$ del hematocrito en los pacientes sometidos a mini abdomen de Juárez Avelar.

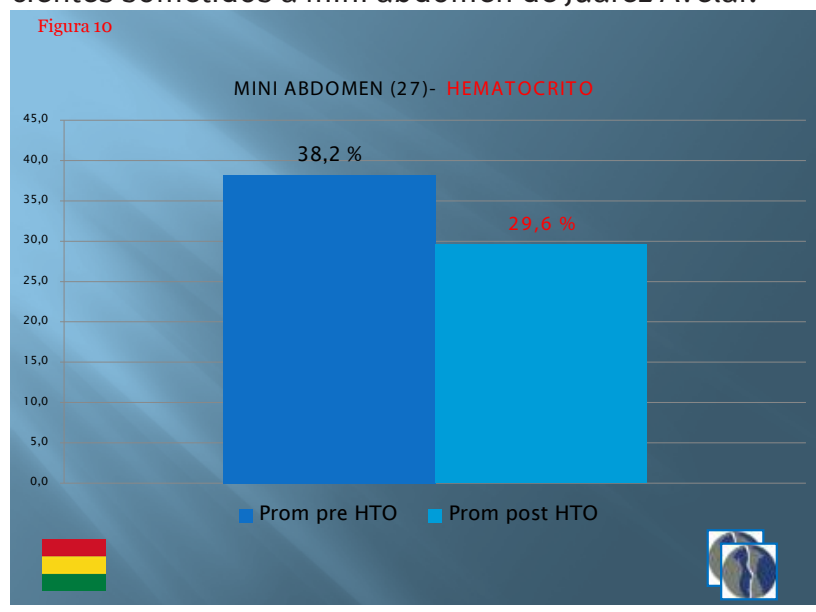

Figura 11, finalmente observamos que la hemoglobina en grupo del mini abdomen de la misma manera que en las gráficas anteriores se comporta de manera isoeléctrica en los controles pre operatorios pero la línea tendencial de color rojo sobre las líneas verdes nos muestra un franco descenso que inicia en $10 \mathrm{grs} / \mathrm{dls}$ a niveles cercanos de $9 \mathrm{grs} / \mathrm{dls}$.

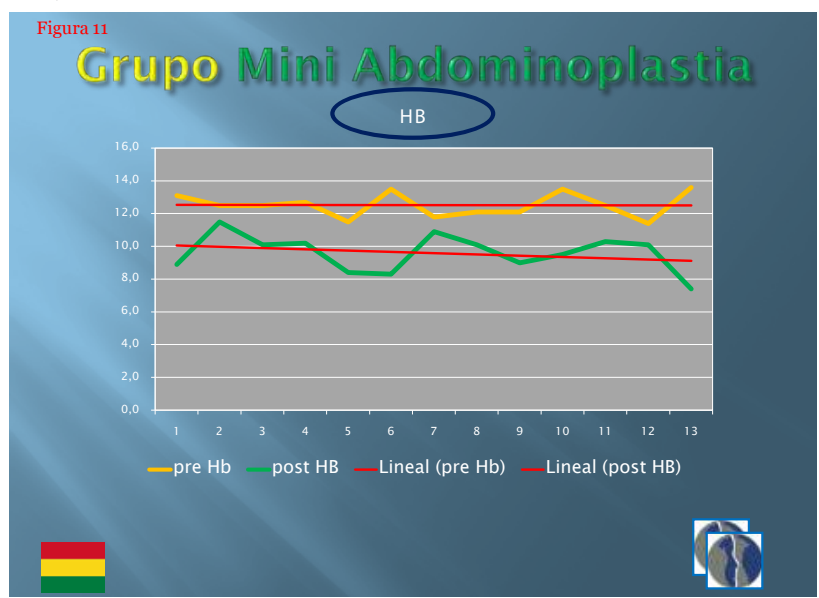


Y si lo transformamos nuevamente a barras, la figura $12 \mathrm{co-}$ rrespondiente al grupo control de los pacientes sometidos a mini abdominolipoplastía de Juárez Avelar, objetivamente nos señala un descenso de los valores Pre operatorios de $12,5 \mathrm{grs} / \mathrm{dls}$ a niveles medidos entre el zer y el 5 to día de $9.6 \mathrm{grs} / \mathrm{dls}$ para los pacientes post operados en este grupo.

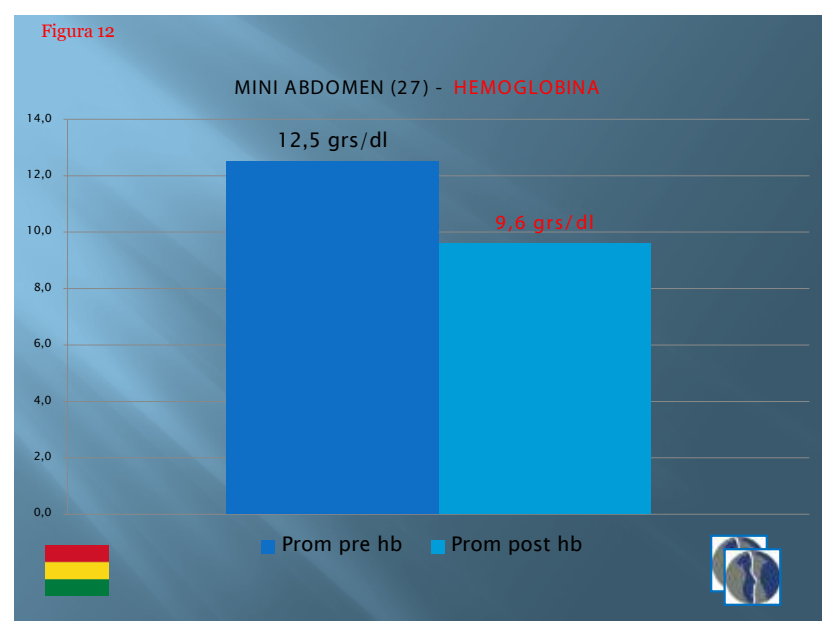

Las siguientes imágenes 13 y 14 nos señalan tal vez los resultados más objetivos del presente estudio.
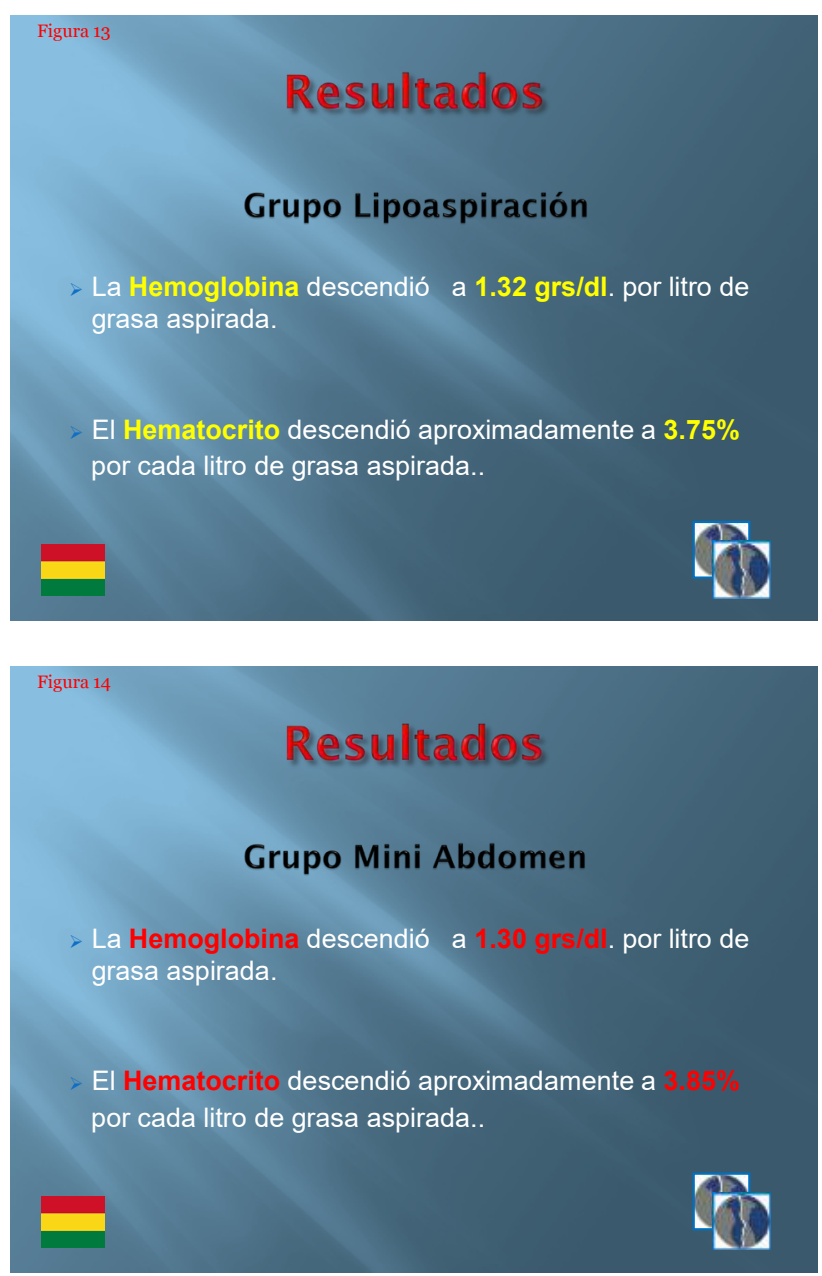

Observamos también que no existe diferencia significativa en los resultados entre ambas técnicas empleadas.

\section{Discusión.}

La medición del hematocrito y la hemoglobina son determinantes a la hora de definir el volumen a lipoaspirar...?

\section{Conclusiones.}

La determinación de la Hemoglobina y del Hematocrito NO es un método exacto para definir el volumen a lipoaspirar en cada paciente, sin embargo nos puede guiar y ayudar mucho para tener en cuenta de acuerdo al volumen aspirado los posibles niveles de descenso de la $\mathrm{Hb}$ y $\mathrm{Hto}$ en el post operatorio.(13).

El Peso del paciente es el factor más importante en la estimativa del valor probable a lipoaspirar.

Mantener y respetar hasta el $5 \%$ de límite en relación al peso corporal es fundamental. (13).

No presentamos pacientes con complicaciones clínicas o descensos de la Hemoglobina menores a 7.5grs/dls. que precisaran hemo transfusión en ambos grupos operados.

Los pacientes cuyos volúmenes aspirados fueron mayores a $2500 \mathrm{mls}$ de grasa, presentaron en el post operatorio quejas como fatiga, cefalea intermitente por 5 días, astenia, hipotensión ortostática leve en los 3 primeros días , taquicardia y cansancio fácil, así como edema importante de áreas lipoaspiradas.

No aconsejamos la retirada de grandes volúmenes de grasa mayores a 3 litros, ya que pasadas estas cifras los signos clínicos de anemia aguda y los riesgos de descompensación aumentan en los pacientes, a no ser que se desee realizar transfusiones o auto transfusiones sanguíneas durante el trans o post operatorio. Esta conclusión es realmente valedera para nosotros por que creemos que una cirugia plástica estética es una cirugía programada y no deberíamos preocuparnos por rehabilitar y compensar a nuestros pacientes del punto de vista hemodinámico transfundiendo ningún fluido o componente sanguíneo en el post operatorio que no sea unicamente aquellos necesarios como soluciones isotónicas para lograr esta estabilización además de los riesgos ineherentes y propios de cualquier hemotransfusión.

A pesar de los conceptos claros e indicaciones precisas de la Asociación Americana de Cirugía Cosmética con respecto a los riesgos de una megalipoescultura y al límite para la extracción de grasa, particularmente nuestro servicio no realiza este procedimiento por considerar que existen otros métodos hoy en día más efectivos para la pérdida de peso. (12). 
No olvidemos que la lipoaspiración es un método quirúrgico programado para modelar la silueta corporal y no para perder peso... reitero! Siendo innecesario correr mayores riesgos a no ser los propios correspondientes para cada intervención mencionada.

\section{Bibliografía.}

1. Avelar, J.M.: uma nova técnica de Abdominoplastia Sistema vascular fechado de rethalho subdermico dobrado sobre si mesmo combinado com lipoaspiracao. Rev. Brasileira de Cirurgia. N. 13, 88/89,(1/6),3-20. Nov/Dec 1999.

2. Avelar,J.M.: Abdominoplasty Without Panniculus Undermining and Resection: analysis and 3- year Follow up of 97 Consecutive Cases. Aesth.Surg.J.Pp. 16- 25, Jan/ Feb.2002.

3. Avelar,J.M.: Abdominoplastia sin despegamiento y sin resección del panículo. Cirugía Plástica Reconstructiva y Estética. Dr. Felipe Coiffman. Tomo IV, Tercera Edición. Cap. 345. 3325 -3337. 2008.

4. American Society for Plastic Surgery. Information on Stastics. Available at www. Plastic surgery.org. Accessed March, 2015.

5. Hetter GP. Blood and fluid replacement for lipoplasty procedures. Clin Plastic Surg.1989, 16:245-8.

6. Illouz. Y.G. Body contouring By lipolysis: 5 -year experience with over 3000 cases.Plast. Reconstr. Surg. 72: 591-597, 1983.

7. Iverson RE, Lynch MD, and the ASPS Commitee on Patient Safety. Practice advisory on Liposuction. Plast Reconstr Surg. 113: 1478-1485. 2004.

8. Klein J.A. The tumenscent technique for Liposuction Surgery. Am J Cosmetic Surg.; 4: 263. 1987.

9. Rem-Yeu tsai et all. Evaluation of blood loss during Tumescent Liposuction in Orientals. Dermatologic surg. 24: 1326-1329. 1998.

10. Suárez Oyhamburú, D.L.: Control de la hemoglobina sanguínea y del hematocrito en pacientes sometidos a lipoescultura y mini abdominolipoplastía con la técnica de Juárez Avelar. IX Congreso Bolivariano de Cirugía Plástica de la FILACP. Santa Cruz de la Sierra, Bolivia. Septiembre, 2015.

11. Suárez Oyhamburú, D.L.: Control de la hemoglobina sanguínea y del hematocrito en pacientes sometidos a li- poescultura y mini abdominolipoplastía con la técnica de Juárez Avelar. XI Congreso Boliviano de Cirugía Plástica Estética y Reconstructiva. Cochabamba, Bolivia. Marzo, 2014.

12. Ulmar H. Choundry, et all. The importance of Preoperative Hemoglobine Evaluation in Large Volume Liposuction. Lessons learned from Our 15-year Experience. Annals of plastic surgery. Volume 61, N 3, September 2008.

13. Fernández Sanza et all, Megaliposucción: estudio de 120 casos. Revista de la FILACP, Volumen 33. N 1. Enero, febrero, marzo, 2007.

\section{Dirección del Autor.}

Dr. Darío Lautaro Suárez Oyhamburú. Calle Manuel Ignacio Salvatierra, 466. Entre Potosí y Tarija. Edificio E \& E. Bloque B, depto. 2. Planta baja. Santa Cruz de la Sierra, Bolivia. Email: dariocirujano@yahoo.com.ar WS: 59170948888.

\section{Dirección de la Co -autora.}

Dra. Jessika Verónica Schaymann Lora.

Calle René Moreno, 667.

Clínica Médica Siraní.

Santa Cruz de la Sierra, Bolivia.

Email: jschaymann@clinicasirani.com 